\title{
Un modèle économique et social pour la conservation de la biodiversité : des filières oléagineuses durables issues de produits forestiers non ligneux (PFNL) pour préserver la Réserve de Faune du Dja (RFD) au Cameroun
}

\author{
Emmanuelle Roques ${ }^{1, *}$, Cécile Lachaux ${ }^{1}$, Théo Tournebize ${ }^{1}$, Aimé Manfred Epanda ${ }^{2}$ \\ et Miranda Mikam Akongongol ${ }^{3}$ \\ ${ }^{1}$ Man and Nature, 16 rue d'Alsace, 68600 Algolsheim, France \\ 2 Tropical Forest and Rural Development, Yaoundé, Cameroun (Centre) \\ 3 Appui à l'Autopromotion de la Femme de la Boumba et de Ngoko, Lomié, Cameroun (Centre)
}

Reçu le 13 novembre 2018 - Accepté le 31 décembre 2018

\begin{abstract}
Résumé - Cet article présente un modèle socioéconomique innovant mis en place en périphérie d'une zone à haute biodiversité : la Réserve de Faune du Dja (RFD), au Cameroun. Les produits forestiers non ligneux (PFNL) de la RFD sont depuis toujours utilisés par les populations locales pour des mets ou remèdes traditionnels. L'objectif de ce projet collaboratif associatif/privé a été de structurer les filières PFNL, afin que les populations bénéficiaires, en particulier les femmes, puissent en tirer les revenus nécessaires à leur développement. Grâce à cette alternative au braconnage, les communautés locales trouvent un intérêt économique à protéger et gérer durablement la forêt.
\end{abstract}

Mots clés : Cameroun / biodiversité / développement durable / produits forestiers non ligneux / cacao / Réserve de Faune du Dja / chaînes de valeur / filières oléagineuses durables / huiles végétales / société civile / organisation non gouvernementale

\begin{abstract}
A social and economic model to protect biodiversity: sustainable oilseeds value chains from Non-Timber Forest Products (NTFP) to protect the Dja Faunal Reserve (DFR) in Cameroon. This paper presents a socioeconomic model, implemented in surrounded areas of a high biodiversity hotspot: the Dja Faunal Reserve (DFR), in Cameroon. Non-Timber Forest Products (NTFP) of DFR have always been used by local people for food or traditional medicine. This associative/private project has aimed at structuring the NTFP value chains, in order to ensure the beneficiary communities, particularly women, to earn the revenues necessary to their development. With this alternative to poaching, local communities find it economically worthwhile to protect and sustainably manage the forest.
\end{abstract}

Keywords: Cameroon / biodiversity / sustainable development / Non-Timber Forest Products / cocoa / Dja Faunal Reserve / value chains / sustainable oilseeds value chains / vegetable oils / civil society / non-governmental organization

\section{Introduction}

Le bassin du Congo est une zone de forêts tropicales humides qui abritent la biodiversité la plus riche d'Afrique. Parmi les espèces fauniques et floristiques, on compte plus de 400 espèces de mammifères, 1000 espèces d'oiseaux et

\footnotetext{
*Correspondance : rques.emmanuelle@gmail.com
}

vraisemblablement plus de 10000 espèces de plantes dont environ 3000 endémiques (Congo Basin Forest Partnership, 2005). Depuis plusieurs décennies, une cinquantaine d'aires protégées ont été créées dans cette zone, réparties sur cinq pays d'Afrique centrale: Congo, Cameroun, Gabon, République Démocratique du Congo et République d'Afrique Centrale.

Avec ses 526000 ha, couvrant $18 \%$ du réseau national des aires protégées, la Réserve de Faune du Dja (RFD) est la plus grande aire protégée du Cameroun (Fig. 1). Elle est située dans 


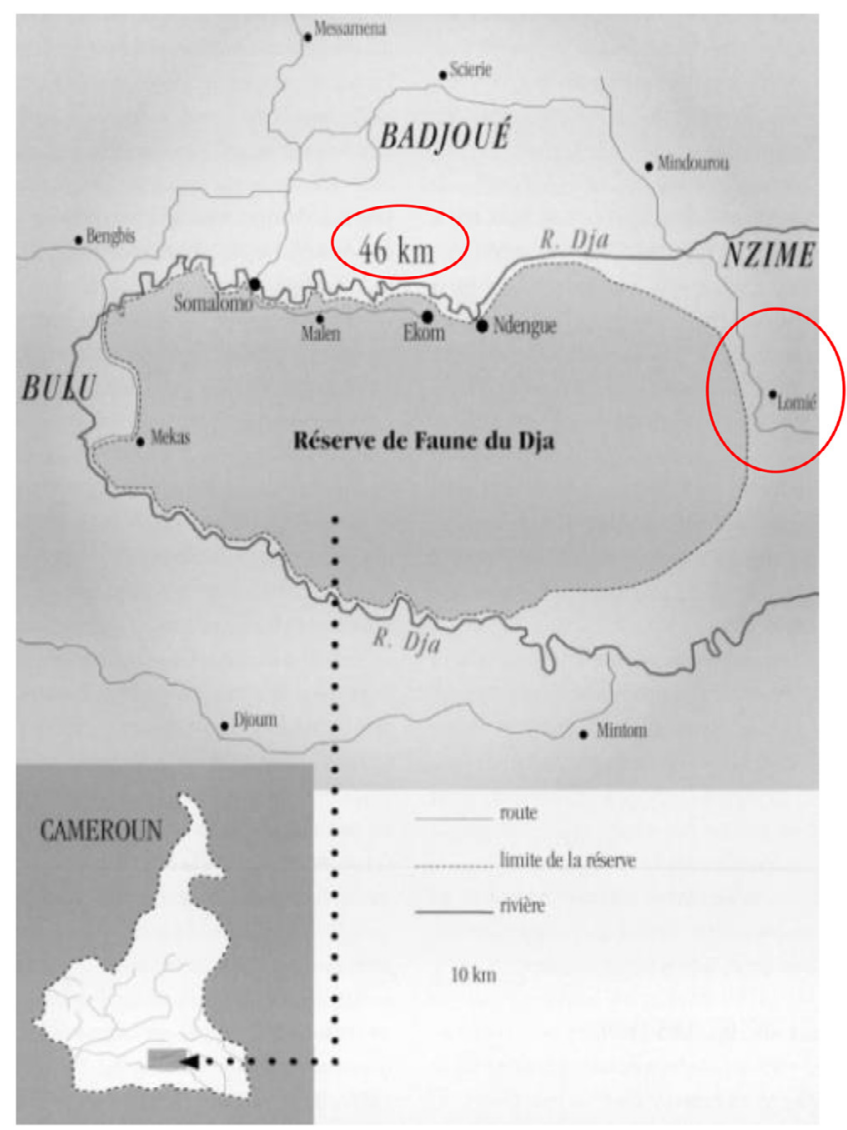

Fig. 1. Cameroun, Réserve de Biosphère du Dja et zones d'intervention.

la partie méridionale du Cameroun, à cheval entre les régions de l'Est et du Sud, dans les départements du Haut Nyong et Dja-et-Lobo. Créée en 1950, et inscrite sur la Liste du patrimoine mondial en 1987, elle est aujourd'hui sous la tutelle de la Direction de la Faune et des Aires Protégées (DFAP) du Ministère des Forêts et de la Faune (MINFOF). Délimitée par le fleuve Dja sur les 2/3 de son périmètre, la RFD possède une diversité biologique exceptionnelle: elle accueille plus de 100 espèces de mammifères, dont 17 espèces de primates (gorille de plaine, chimpanzé, mangabey à collier blanc...).

Toutefois, la réserve est menacée par des activités d'extraction minière (cobalt, nickel, or, diamants), des plantations d'hévéa, de grands chantiers d'infrastructures en cours de réalisation (barrage, chemin de fer), et des exploitations forestières dans les zones ouest, sud et est (Diedhiou et Diawara, 2015). À cela s'ajoute l'augmentation de la population en périphérie de la RFD provoquant notamment l'avancée du front agricole et des exploitations illégales de produits forestiers. Enfin surtout, le braconnage représente une pression directe très inquiétante qu'il est difficile de combattre avec des actions de répression, les administrations de la protection étant hélas inefficaces, parfois corrompues.

Historiquement, les programmes environnementaux de protection de la biodiversité se sont articulés autour de la restriction d'accès aux aires protégées via des mesures de surveillance et de répression, au détriment des populations locales vivant en périphérie (McNeely, 1994). En niant leurs compétences dans l'usage de ces espaces, ce type d'approche les spolie de leur espace de vie et les prive alors d'une grosse part de leur revenu. Pire encore, ces modèles répressifs peuvent provoquer de véritables conflits État/populations, comme cela a été le cas en 2000 lors de la mise en place du sanctuaire de Mengame, au Sud Cameroun (Assembe Mvondo et Lema Ngono, 2007).

De fait, l'approche de conservation classique peut difficilement apporter des solutions pérennes dans ces zones. Il semble alors que les solutions durables de protection de la biodiversité doivent être mises en place à l'échelle locale, en impliquant les communautés vivant dans et autour de ces zones protégées. Grâce à des alternatives économiques au braconnage et à l'agriculture expansive, l'objectif est d'amener les populations à soutenir l'existence des zones protégées et à adopter un mode de gestion durable de ces espaces.

C'est dans ce contexte que Man and Nature ${ }^{1}$ et ses partenaires ont voulu proposer un modèle économique innovant de conservation de la biodiversité, basé sur la mise en place de filières oléagineuses durables, en périphérie de la RFD au Cameroun. En structurant des chaînes de valeur sur les produits forestiers non ligneux oléagineux, le projet a permis l'implémentation d'un véritable modèle économique local, dont les enjeux, problématiques et impacts socio-économiques feront l'objet de cet article.

\section{Produits d'intérêt et méthodes}

\subsection{Les produits forestiers non ligneux (PFNL)}

Les PFNL sont définis par l'Organisation des Nations Unies pour l'Agriculture et l'Alimentation (FAO) comme des produits d'origine biologique (faune et flore) autres que le bois, provenant de la forêt naturelle, artificielle ou des systèmes agroforestiers (CBFP, 2005).

Tous les ans, la forêt camerounaise produirait plus de 1000 tonnes (feuilles, écorces, graines, etc.) de PFNL végétaux (Ngome-tata, 2006). Traditionnellement, leur usage peut être alimentaire, médicinal, cosmétique ou rituel.

Les études menées par la FAO pour le renforcement de la sécurité alimentaire en Afrique Centrale (2005-2008) ont révélé que le secteur des PFNL représente un potentiel de développement considérable pour les communautés locales. Il joue en effet un rôle socioéconomique essentiel, notamment pour les femmes, car la vente de produits bruts ou transformés créent des revenus significatifs pour de nombreux ménages, surtout en période de soudure (COMIFAC, 2013). La plupart de ces produits sont pourtant commercialisés dans le secteur informel avec des acteurs par filière très peu organisés, faute de réglementation et cadre institutionnel clair dans ce domaine (CBFP, 2005).

Cela représente en outre une véritable opportunité de développement pour les populations pygmées Baka de la RFD. En effet, elles possèdent un savoir immense sur les PFNL, qu'elles consomment abondamment, mais n'en tirent quasiment aucun revenu. Souvent, elles troquent le fruit de leur collecte aux populations Bantoues contre des produits de première nécessité (Ndzomo Abanda, 2008).

\footnotetext{
${ }^{1}$ Pour plus d'informations, consulter: www.manandnature.org.
} 


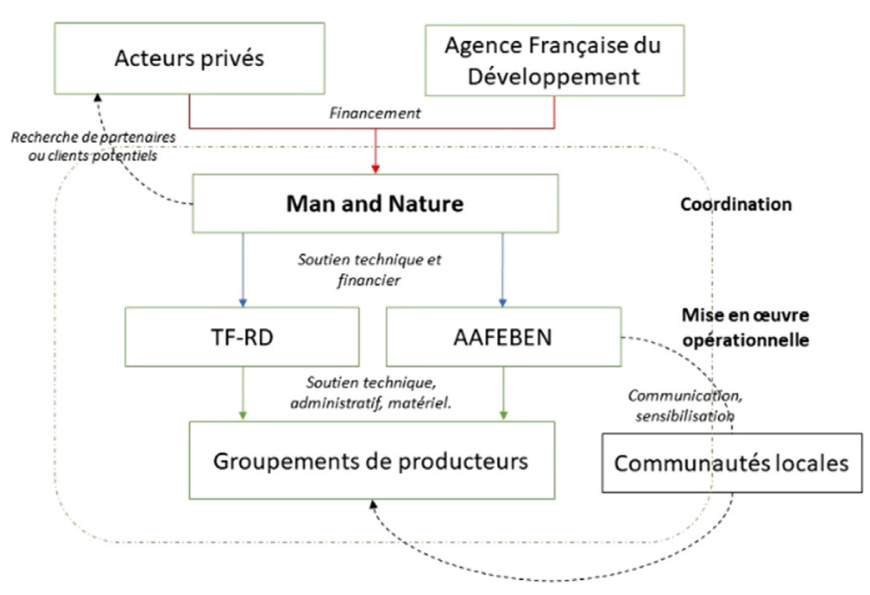

Fig. 2. Mise en œuvre du projet aux 4 niveaux d'action.

\subsubsection{Le cas particulier du cacao}

Depuis le début du $20^{\mathrm{e}}$ siècle, la cacaoculture est pratiquée en forêt, sur des parcelles de petites dimensions pour assurer un ombrage léger aux jeunes cacaoyers (Kwesseu Petguen, 2010). Suite à la crise du cacao à la fin des années 1980, l'extension des cultures vivrières s'est traduit par une déforestation accrue mais également par la diversification des cultures à l'intérieur des cacaoyères. En particulier, les systèmes agroforestiers montrent des performances productives très acceptables, alors qu'ils demandent peu d'intrants, peu de technologies, tout en étant moins sensibles aux variations climatiques (Arizmendi Gonzalez, 2013).

D'après la définition de la FAO rappelée précédemment, les fèves de cacao seront considérées dans le document comme des PFNL, car issues d'un système agroforestier.

\subsubsection{Justification du choix de ces filières}

Les PFNL valorisés dans ce projet ont été choisis pour leurs propriétés lipidiques mais aussi leur importance culturelle, leur intérêt cosmétique et alimentaire, et bien entendu leur potentiel de rentabilité. Il s'agit entre autres des amandes de "moabi» (Baillonella toxisperma), de mangue sauvage (Irvingia gabonensis), d' «allanblackia » (Allanblackia floribunda), de «njansang» (Ricinodendron heudelotii), de «mbalaka» (Pentaclethra macrophylla) et des fèves de cacao (Theobroma cacao). Certains de ces produits étaient déjà exploités dans la zone, soit consommés, soit rachetés directement par des grossistes avec toutefois une très faible valeur ajoutée. D'autres étaient connus des populations mais n'étaient pas valorisés, par méconnaissance du marché-ou faute de débouchés.

Outre leur fort ancrage culturel, les ressources représentent un enjeu écologique. Le moabi, par exemple, arbre majestueux de la famille des Sapotaceae, est l'une des espèces les plus endémiques des forêts tropicales du Bassin du Congo. Hélas, à cause de l'exploitation intensive de son bois, et de la disparition progressive des éléphants, principaux propagateurs de ses graines, il est dorénavant classé «vulnérable» par l'Union internationale pour la conservation de la nature (IUCN).

\subsection{Mise en œuvre, acteurs et bénéficiaires du projet}

Le modèle économique des filières PFNL s'intègre dans un projet de grande envergure, mis en place par Man and Nature et financé par l'Agence française du developpement (AFD) sur 9 filières économiques issues de ressources naturelles dans des zones à haute biodiversité ${ }^{2}$. Ce programme est déployé avec 5 Organisations de la Société Civile (OSC) au Cameroun (4) et au Sénégal (1) impliquant directement 44 groupements de producteurs - soit plus de 3500 au total, répartis sur 5 zones de forte biodiversité, dont la RFD. Le projet est ainsi mené à 3 niveaux d'actions et implique 4 types d'acteurs selon leur rôle de bailleur, de coordinateur, d'agent opérationnel ou de producteur-bénéficiaire ( $c f$. Fig. 2).

En ce qui concerne les PFNL, les zones d'intervention se situent au Nord et à l'Est de la RFD, où les OSC camerounaises $\mathrm{TF}^{-\mathrm{RD}^{3}}$ (Tropical Forest and Rural Development) et AAFE$\mathrm{BEN}^{4}$ (appui à l'autopromotion de la femme de la Boumba et de Ngoko) agissent depuis des années pour le développement rural. Concrètement, elles appuient les producteurs à se structurer en unités de production en les formant sur les techniques de production/collecte, de transformation, de commercialisation et sur la gestion administrative et financière des coopératives. Elles jouent aussi le rôle d'intermédiaire auprès des institutions officielles ou autorités coutumières (chefs de village), qu'il est essentiel d'intégrer à l'approche.

Ainsi, les bénéficiaires du projet sont nombreux et augmentent chaque année :

- au Nord de la RFD, 160 cacaoculteurs sont regroupés autour d'une coopérative : la SOCOPROCAPDJA (Société cooperative des producteurs de cacao du Dja) et 8 groupements d'initiative commune (GIC) de collectrices de PFNL ont été structurés et légalisés;

- à l'Est de la RFD, la coopérative le Centre Vert de Lomié regroupe en tout près de $500 \mathrm{femmes}$ autour de la valorisation des PFNL.

\section{Des chaînes de valeurs durables}

\subsection{La structuration des filières}

\subsubsection{Organisation de la récolte au niveau du terrain}

Les communautés collectent les PFNL selon leurs pratiques traditionnelles. Rassemblées en groupe (1 ou 2 par village), elles organisent des campagnes de récolte, celles-ci s'accompagnant parfois de chants ou de rituels.

Grâce au projet, les groupes de producteurs ont pu bénéficier de formations aux techniques de collecte garantissant des produits de bonne qualité et dans le respect de la ressource exploitée.

En particulier, plus de la moitié des producteurs de cacao répondent depuis 2016 aux standards de durabilité du référentiel Rainforest Alliance. Les cacaoculteurs ont ainsi nettement diminué l'emploi de produits phytosanitaires, au

\footnotetext{
${ }^{2}$ Pour plus d'informations sur ce projet: http://www.manandnature. org/projets/afd/.

${ }^{3}$ Site internet de TF-RD : http://www.tropicalforest-rd.org/.

${ }^{4}$ Pages internet de présentation des ONG : http://www.manandnature. org/partenaires/ong/.
} 
profit d'alternatives plus vertes, telles que des barrières végétatives (The Rainforest Alliance, 2017).

\subsubsection{Première transformation}

Les produits sont ensuite centralisés à la Base Vie de TF$\mathrm{RD}$ à Kabilone (BVK, voir Encadré 1$)$ ou le Centre Vert de Lomié (CVL). Les sacs sont pesés et tracés (origine, date de collecte, numéro de lot...).

\section{Encadré 1. La Base Vie de Kabilone: une véritable vitrine du développement durable dans la RFD.}

Outre sa fonction de point focal du projet sur le terrain (magasin de stockage, lieu de vie des techniciens, lieu des transactions...) la Base Vie de Kabilone est un véritable site écotouristique. En effet, 8 lodges modernes, une salle de conférence, un magasin, un bureau et un restaurant ont été construits afin d'accueillir des touristes amoureux de la nature et sensibles aux problématiques de conservation. En 2017, une douzaine de membres de la Fondation Maison du Monde y ont séjourné et ont pu découvrir les ressources valorisées par le projet sur un sentier botanique mis en place autour de la base.

À terme, ce lieu vise à devenir un Centre de diffusion, de documentation et de recherche pour la conservation et le développement (projet «CERCOD»).

Certains produits sont directement stockés dans un magasin (à l'abri de la lumière), d'autres vont subir un second séchage, dans un séchoir à bois.

Ces séchoirs ont été construits par les OSC locales en 2016, en s'inspirant du modèle FAO de séchoir statique avec générateur d'air chaud à bois et échangeur air/air ( $c f$. Fig. 3). Les produits à sécher sont disposés au-dessus du foyer sur une natte en bambou. La capacité du dispositif permettant le séchage de plus d'une tonne de produits à la fois, il est particulièrement adapté aux contraintes météorologiques de la période de collecte des PFNL, de juillet à décembre, concomitante avec la saison des pluies.

À l'issue du séchage, les produits, regroupés par lot selon le village et la zone de collecte, sont prêts à être vendus. Et c'est à ce niveau que réside une des grandes forces du projet: avec les ventes groupées, les communautés productrices possèdent un pouvoir de négociation, grâce à une structure de transaction, à des produits en grande quantité et de bonne qualité. En une seule vente, $500 \mathrm{~kg}$ à $1 \mathrm{t}$ de produits peuvent être écoulés, pouvant ainsi rapporter jusqu'à 1 million FCFA (soit $1500 €$ ) à la coopérative.

Une partie significative (surtout pour la zone Est) est achetée à ce niveau par des grossistes, essentiellement Nigérians, sans subir de $2^{\mathrm{e}}$ transformation. L'autre partie est rachetée à la coopérative par TF-RD ou AAFEBEN qui vont prendre en charge la seconde transformation des produits.

\subsubsection{Seconde transformation}

La plate-forme de Yaoundé transforme les PFNL en huiles/ beurres végétaux et en poudres alimentaires après broyage des tourteaux.

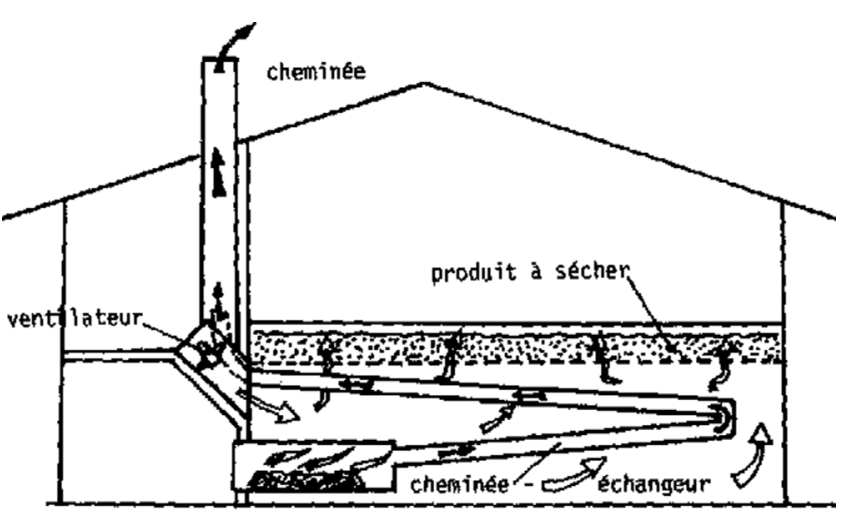

Vue en coupe

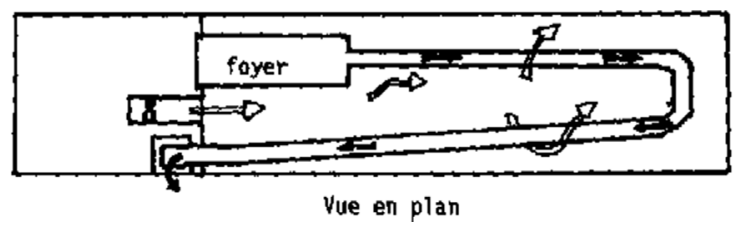

Fig. 3. Schéma d'un séchoir statique avec générateur d'air chaud à bois et échangeur air/air (D'après Cruz et al., 1988).

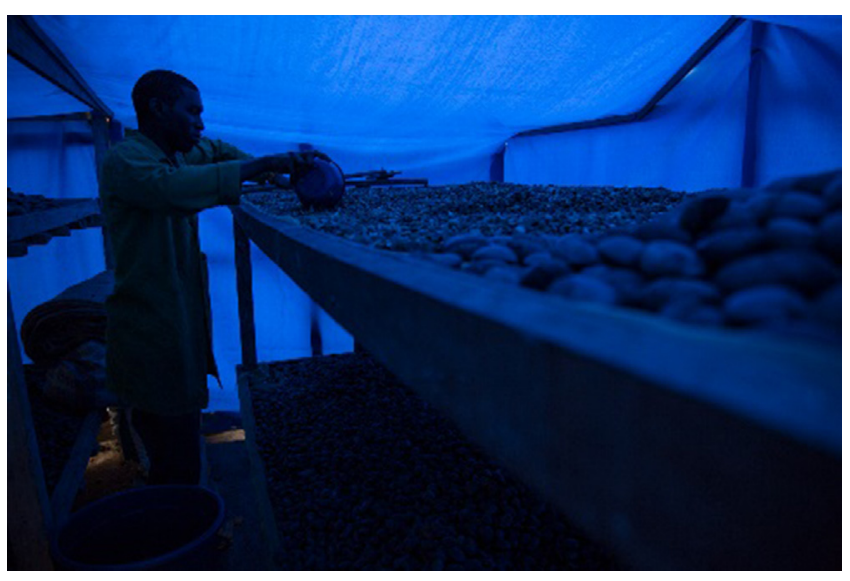

Fig. 4. Noix de moabi étalées sur les claies du séchoir solaire de la plate-forme de transformation de Yaoundé.

S'ils ne sont pas pressés tout de suite, les PFNL sont disposés sur des claies dans un séchoir solaire, à l'abri des nuisibles et protégés de la lumière grâce à des bâches opaques (cf. Fig. 4). Le taux d'humidité des produits finit alors de décroître et se stabilise avant le pressage. Puis, la trituration est réalisée à froid à l'aide de l'extrudeuse d'huiles naturelles vierges (modèle KOMET DD 85 G, $c f$. Fig. 5). Enfin, les plus grosses impuretés sont éliminées via une simple filtration gravitationnelle sur tamis (pores $0,1-0,3 \mathrm{~mm}$ ).

Les coproduits sont également valorisés : les tourteaux sont broyés en poudre pour un usage alimentaire (mangue sauvage, njansang, cacao) tandis que les «pâtes » (tourteaux très gras issus d'une séparation partielle) sont utilisées pour une fabrication artisanale de savons. 


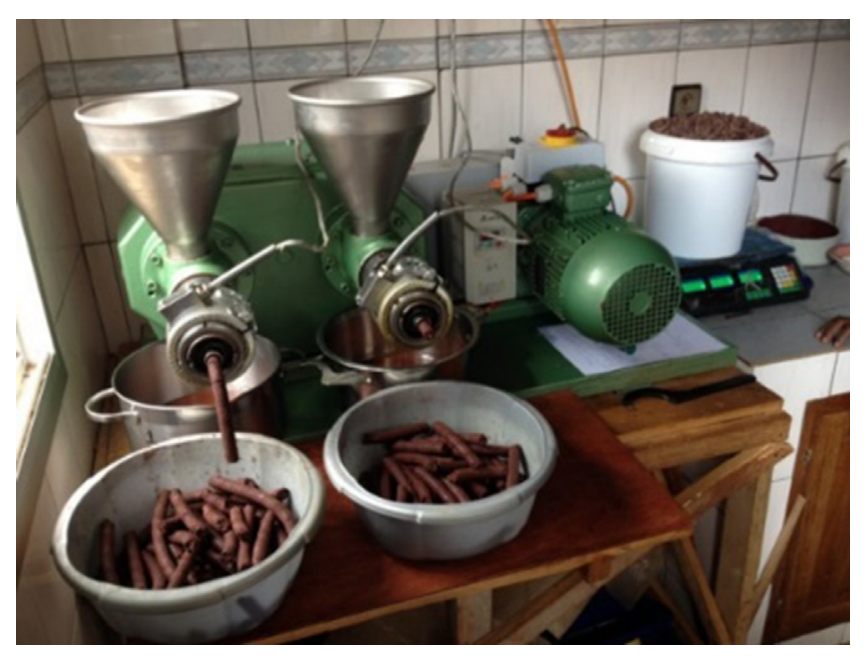

Fig. 5. Pressage des fèves de cacao dans l'extrudeuse KOMET DD 85-G. Laboratoire de pressage de Yaoundé.

Le tourteau toxique de moabi a, quant à lui, fait l'objet de recherches avec l'IRAD (Institut de recherche en agronomie et développement) pour être utilisé comme biopesticide, notamment pour les capsides du cacao.

C'est donc une transformation totale et propre: elle nécessite très peu d'intrants, valorise tous les coproduits et ne génère pratiquement aucun déchet non biodégradable.

\subsection{Une meilleure connaissance des produits et des procédés}

En plus des activités opérationnelles, une partie du programme de Man and Nature a été dédié à la construction d'une expertise autour des produits et des procédés de transformation.

Grâce à un partenariat avec les Huileries Bertin, les OSC locales ont pu bénéficier d'une formation sur les huiles végétales et leurs propriétés organoleptiques. À cela se sont ajoutés d'autres mécénats de compétences avec des structures privées (Sisley, Mane), académiques (Université de Yaoundé I, INP Toulouse), institutionnelles (CIRAD, IRD) ou des laboratoires d'analyses (Centre Pasteur de Yaoundé).

Les opérateurs du projet ont ainsi acquis une meilleure compréhension de leurs produits. Ils ont pu cibler et optimiser les paramètres influençant la qualité des produits (notamment les facteurs d'oxydation tout au long de la chaîne de valeur). Un mini-laboratoire d'analyse a d'ailleurs été mis en place à la plate-forme de Yaoundé, afin de mesurer sur place les indices d'acide et de peroxyde des huiles.

\subsection{La commercialisation}

\subsubsection{Société de commercialisation}

L'entreprise sociale Tropical Forest Food and Cosmetics (TF-FC) a été créée en 2016 afin de mener les transactions commerciales locales et internationales. Basée à Yaoundé, les activités de l'entreprise regroupent la production, transformation et distribution des PFNL. TF-FC peut donc soit acheter la matière première aux coopératives afin d'en assurer sa vente sous forme brute ou transformée, ou bien seulement proposer un service de transformation des PFNL (comprenant le séchage solaire, le pressage et le conditionnement) aux coopératives qui veulent réaliser elles-mêmes la vente de leurs produits.

\subsubsection{Coûts de production et de revient}

Les coûts de production sont essentiellement liés aux coûts d'approvisionnement qui comprennent:

- des charges variables : achat de la matière première à la coopérative (avec transport jusqu'au point focal) et transport jusqu'à Yaoundé ;

- des charges fixes liées aux activités de l'OSC sur le terrain : salaires des opérationnels, fonctionnement (repas, transport...), amortissement du matériel et du mobilier...

Il faut à cela ajouter les coûts de seconde transformation (forfait journalier englobant les coûts de main d'œuvre, fonctionnement, maintenance des appareils, amortissements...) ainsi que les coûts de distribution, (packaging, livraison, outils de marketing...), pour enfin obtenir le coût de revient.

Pour atténuer les coûts de production et de revient, la marge de manœuvre réside essentiellement dans la réduction des charges fixes. De ce fait, la minimisation des pertes au niveau du terrain et l'optimisation du rendement de transformation représentent de véritables opportunités pour la rentabilité des chaînes de valeur.

\subsubsection{Stratégie commerciale}

\subsubsection{Prix de vente}

La finalité du projet est l'amélioration des revenus des producteurs sur le terrain. Or, un prix d'achat des matières premières trop élevé entraînerait un prix de vente peu compétitif sur le marché et limiterait les quantités vendues. L'enjeu est donc de trouver le meilleur compromis entre équitabilité auprès des producteurs, rentabilité de la filière, et attractivité pour écouler suffisamment de volume et implanter l'approche aux échelles locale et sous-régionale. L'objectif in fine étant de stimuler la production sur le terrain par la demande du marché.

Des prix de vente ont été fixés à partir du calcul des coûts de revient (cf. Sect. 3.3.2) et de l'étude de la concurrence. La valeur ajoutée, correspondant à la différence entre les prix de vente et les coûts de production, est de l'ordre de $10 € / \mathrm{kg}$ d'huile ( $c f$. Tab. 1). Les bénéfices générés grâce à elle sont majoritairement réinvestis dans l'achat de nouvelles matières premières aux communautés. Ils permettent en outre à TF-FC d'entretenir son activité de production et de vente (acquisition de ressources matérielles, création de fonds de roulement...).

\subsubsection{2 Études de marché}

En 2016, une enquête préliminaire a été menée dans les villes de Yaoundé et Douala auprès de professionnels de la cosmétique (salons d'esthétique et de massage) pour les produits transformés afin d'identifier la demande du marché camerounais et les offres concurrentes déjà disponibles - prix, qualité, capacités d'approvisionnement, packaging... En 
Tableau 1. Prix de vente et valeur ajoutée des produits bruts et transformés.

\begin{tabular}{lllr}
\hline PFNL & Prix /kg de produit brut $(€)$ & Prix / kg d'huile $(€)$ & Valeur ajoutée $(€)$ \\
\hline Moabi & 0,38 & 18,29 & 8,8 \\
Cacao & 2,13 & 27,44 & 14,1 \\
Mangue sauvage & 1,52 & 18,29 & 10,3 \\
Njansang & 2,29 & 27,44 & 11,5 \\
Mbalaka & 0,87 & 18,29 & 9,5 \\
\hline
\end{tabular}

parallèle, une étude sur les différents référentiels et labels d'éco-responsabilité a été réalisée. Les conclusions ont révélé que, parmi les PFNL transformés, seul le beurre de cacao est présent sur le marché cosmétique. Par ailleurs, les beurres végétaux vendus au Cameroun (avocat, cacao, karité...) sont rarement purs, parfois coupés avec de l'huile de palme et pour la plupart mélangés avec des parfums artificiels. Les produits développés sont ainsi plutôt destinés à des consommateurs consciencieux, qui s'intéressent non seulement aux produits naturels de qualité mais aussi à la démarche d'innovation et de conservation. Pour ces raisons, la certification biologique ou équitable des filières PFNL (hors cacao), n'a pas été jugée pertinente dans cette première phase de projet. En effet, l'obtention d'un tel label, coûteux et nécessitant un lourd suivi, n'apporterait pas de réelle valeur ajoutée aux produits, déjà innovants.

Une étude de marché à grande échelle est en cours pour analyser le marché transfrontalier Cameroun-Nigéria. Etant le plus gros bassin de consommation de PFNL d'Afrique, le Nigéria joue un rôle incontestable dans la commercialisation des PFNL. L'objectif de l'enquête est de quantifier et qualifier la demande : prix, consommateurs finaux, produits d'intérêt... Elle a aussi pour but de déterminer la forme des produits qui a le meilleur potentiel commercial: matières premières, semitransformées ou huiles végétales. Complétée par une étude du marché interne camerounais, les résultats permettront de connaître la stratégie la plus rentable avec le meilleur partage de bénéfices. Enfin surtout, il est attendu un réel tremplin commercial : gain en visibilité et carnet d'adresses de clients susceptibles de passer des commandes en grandes quantités.

\subsubsection{Acheteurs du nord}

En début de projet, des acheteurs occidentaux du secteur de la cosmétique ont permis de stimuler la production et d'assurer un fond de roulement grâce à des commandes en grandes quantités. En fin 2017, $630 \mathrm{~kg}$ de beurres ont ainsi été vendus à l'international, soit environ un quart de la production.

\subsection{Des filières durables}

En plus des aspects socioéconomiques qui sont évidemment au cœur du projet, il a été également indispensable de maîtriser l'impact environnemental des chaînes de valeur pour s'inscrire dans une démarche complète de développement durable. Le programme de Man and Nature comporte ainsi un important volet sur le maintien des ressources exploitées via l'établissement de plans de collecte.

D’une part, plus de 40000 pieds ont été plantés dans des pépinières communautaires, pour être à terme introduits en
Tableau 2. Quantités vendues et revenus au niveau du village.

\begin{tabular}{lcc}
\hline Produit & $\begin{array}{c}\text { Quantité brute achetée } \\
\text { aux communautés }(\mathrm{kg})\end{array}$ & $\begin{array}{l}\text { Revenus totaux } \\
\text { générés }(€)\end{array}$ \\
\hline Moabi & 7160 & 2730 \\
Mangue sauvage & 27680 & 42200 \\
Cacao & 6000 & 10900 \\
Mbalaka & 7300 & 6350 \\
Autres PFNL & 1600 & 1450 \\
\hline
\end{tabular}

forêt. Certains de ces plants ont pour vocation d'enrichir et diversifier les parcelles d'agroforesterie. Ainsi, lorsqu'ils seront en mesure de fructifier dans une dizaine d'années, les producteurs pourront disposer de PFNL directement dans leur plantation.

Il a en outre fallu estimer les conséquences de la collecte sur la régénération de la ressource, en réalisant, pour chaque PFNL exploité, une cartographie et un inventaire floristique géoréférencé ${ }^{5}$.

Dans le cas particulier du cacao en agroforesterie, les méthodes de culture et d'exploitation sont soumises aux standards du label Rainforest Alliance. En plus de définir des modes de gestion écologique des espaces cultivés, la Norme prescrit l'établissement d'un système documenté de management dans lequel les producteurs doivent cartographier et quantifier les capacités de production, et planifier la récolte, similairement aux plans de collecte des produits forestiers sauvages (The Rainforest Alliance, 2017).

\section{Impacts socioéconomiques et environnementaux}

\subsection{Impacts économiques}

Pour la saison 2016, 48,7 t de PFNL bruts ont été achetés au niveau du village ( $c f$. Tab. 2), rapportant au total plus de $63000 €$ aux communautés bénéficiaires. Avec environ 400 producteurs impliqués, les ventes auraient ainsi permis de rapporter en moyenne $160 €$ par producteur, ce qui est un revenu important dans cette zone.

\footnotetext{
${ }^{5}$ À partir des informations récoltées dans cet inventaire, il est possible d'évaluer la capacité de renouvellement de la ressource à partir du calcul du taux de régénération défini selon Rothe (1964) par: $T r=\frac{\text { Nombre } \mathrm{d}^{\prime} \text { individus régénérés }<S(\mathrm{~cm})}{\text { Nombre } \mathrm{d}^{\prime} \text { individus semenciers }>S(\mathrm{~cm})}$, où les individus sont considérés comme des semenciers avec un diamètre à hauteur de poitrine $(\mathrm{dph})>$ seuil $S$ défini $(\mathrm{cm})$ et comme des individus régénérés avec un $\mathrm{dhp}<S(\mathrm{~cm})$. Le seuil est à adapter selon l'espèce et sa morphologie.
} 
Tableau 3. Production d'huile à la plate-forme de transformation de Yaoundé. Saison 2016-2017.

\begin{tabular}{lcccc}
\hline Produit & Quantité d'huile produite $(\mathrm{kg})$ & Valeur de la production $(€)$ & Quantité vendue $(\mathrm{kg})$ & Chiffres des ventes $(€)$ \\
\hline Moabi & 1300 & 27000 & 710 & 18000 \\
Mangue sauvage & 618 & 11300 & 41 & 757 \\
Cacao & 590 & 16200 & 100 & 2740 \\
Autres produits & 212 & 5700 & 158 & 4300 \\
\hline
\end{tabular}

On estime par ailleurs le nombre de bénéficiaires indirects (producteurs et leur famille) autour de 2000 (en moyenne 5 membres par famille).

Pour cette même saison, au niveau de la plate-forme de Yaoundé, $11 \mathrm{t}$ de produits ont été pressés, ce qui a permis de produire environ $2,8 \mathrm{t}$ d'huiles et beurres végétaux pour une valeur de plus de $60000 €$ ( $c f$. Tab. 3).

On note toutefois que, parmi ces 2,4t d'huiles produites, seulement $1 \mathrm{t}$ a été écoulée (42\%), dont environ $700 \mathrm{~kg}$ à l'international. Ainsi localement, uniquement $450 \mathrm{~kg}$ de produits ont été vendus, presqu'exclusivement en petites quantités via des foires ou des marchés. À cela s'ajoutent néanmoins les produits secondaires (poudres et savons), qui, plus connus, rencontrent un certain succès (pour cette même saison, leur vente aurait rapporté environ $3200 €)$.

Pour avoir des impacts pérennes, Man and Nature et ses partenaires souhaitent consolider les débouchés nationaux et sous-régionaux, en s'appuyant, par exemple, sur des revendeurs et/ou enseignes de distribution.

\subsection{Impacts sociaux}

La principale période de collecte étant en juillet-août (saison des pluies), les revenus générés sont extrêmement importants pour la scolarisation des enfants. En effet, l'économie des zone rurales reposant en grande partie sur des activités de subsistance (culture, chasse), les frais de scolarité représentent l'une des dépenses les plus importantes des foyers (Fodouop Kengné, 2003).

Plus concrètement en termes d'éducation, TF-RD a choisi d'investir une partie des revenus issus de la vente du beurre de cacao et des PFNL pour réhabiliter une école à Malen (village proche de Kabilone). Loin d'être un cas isolé en région rurale, l'établissement était hélas à l'abandonfaute de moyens publics alloués à son entretien. TF-RD s'est alors engagé à payer le salaire d'un instituteur pendant une année scolaire, le temps pour les communautés locales de rassembler les ressources nécessaires au fonctionnement de l'établissement. C'est une véritable action de force de la part de TF-RD, qui a permis d'initier un dialogue avec les autorités publiques sur la question de l'isolement rural.

En termes d'entreprenariat et d'émancipation des femmes, les résultats se sont révélés très encourageants. Si les ventes groupées ont permis, d'une part, d'augmenter considérablement leur contribution aux revenus du foyer, les femmes occupent de surcroît des postes à responsabilité dans les groupements structurés, qui sont pour la plupart exclusivement féminins ( $c f$. Tab. 4).

En intégrant les groupements, les femmes Baka ont pu tirer les revenus équitables de leur collecte. Au moins 100 d'entre elles sont membres des coopératives (ou GIC), et certaines occupent même un poste de gestion administrative ou financière. Pourtant, cela semblait inimaginable en début de projet, tant la rivalité avec les ethnies Bantoues est vive dans cette zone (Fodouop Kengné, 2003). Demeurant un cas particulier, il faudra continuer la sensibilisation pour encourager et perpétuer cette nouvelle collaboration.

\subsection{Impacts environnementaux}

Les impacts environnementaux des filières ne sont pas mesurables selon des indicateurs reconnus, tels que les émissions de gaz à effet de serre, par exemple. Une approche rigoureuse d'évaluation environnementale nécessiterait en effet de définir le système d'étude et de réaliser un bilan de tous les intrants et sortants de ce système. L'Analyse de cycle de vie $(\mathrm{ACV})$, notamment, est particulièrement adaptée aux produits biosourcés car c'est la seule méthode multicritère permettant de tenir compte de toutes les étapes de la chaîne de valorisation (AFNOR, 2015). Les ACV réalisées dans le secteur des huiles végétales en France s'appuient sur des inventaires détaillés (appelés ICV), de la culture de la graine oléagineuse jusqu'à la fin de vie de l'emballage (Badey et al., 2017). Néanmoins, compte tenu du contexte géographique et du caractère expérimental du projet, il n'a pas été possible de collecter les données de production avec un tel niveau de détail.

Il a tout de même été possible d'apprécier les bénéfices potentiels sur l'environnement à travers le changement d'habitudes des populations cibles. Des enquêtes effectuées dans la zone de travail en 2017 révèlent que pour environ $80 \%$ des ménages sondés, la vente des PFNL représente désormais la première ou seconde activité génératrice de revenus (avec l'agriculture) ; alors qu'il s'agissait du braconnage en début de projet (Mukam, 2017). Les personnes interrogées se sont également dites prêtes à diversifier les filières PFNL afin de limiter l'impact potentiel sur la régénération de la ressource.

Sur la question du braconnage, des accords environnementaux réciproques ont été signés entre la coopérative du Centre Vert de Lomié, AAFEBEN et un exploitant forestier de la zone, engagé dans la démarche de certification $\mathrm{FSC}^{6}$. En l'échange d'un accès facilité aux zones d'exploitation et la mise à disposition de matériel, les communautés se sont engagées à ne plus pratiquer la chasse dans ces zones. De même, le cahier des charges Rainforest Alliance prescrit formellement le braconnage des espèces menacées (The Rainforest Alliance, 2017).

\footnotetext{
${ }^{6}$ Forest Stewardship Council. Selon la Norme, les entreprises doivent justifier d'un engagement de moyens pour la protection de la faune, en collaboration avec les partenaires locaux (GWZ Wijma Cameroun S. A., 2011).
} 
Tableau 4. Groupement, producteurs et part des femmes impliqués dans les filières PFNL/cacao.

\begin{tabular}{llll}
\hline Produit & Groupements & $\begin{array}{l}\text { Nombre total de producteurs } \\
\text { dans les groupements }\end{array}$ & $\begin{array}{l}\text { Nombre de femmes } \\
\text { dans les groupes }\end{array}$ \\
\hline PFNL hors cacao & $\begin{array}{l}1 \text { coopérative et } 8 \text { groupement } \\
\text { d'initiative commune (GIC } \\
1 \text { coopérative }\end{array}$ & $\begin{array}{l}500 \text { (dont au moins } 100 \\
\text { de l'ethnie Baka) }\end{array}$ & 500 \\
Cacao & 160 & 50 \\
\hline
\end{tabular}

\section{Conclusion}

En s'inscrivant dans une démarche décentralisée de développement durable, le modèle déployé au Cameroun est un exemple accompli de la mobilisation de la société civile pour devenir actrice de son propre développement. Les résultats positifs et l'implication grandissante des communautés semblent valider le succès de l'approche dans les zones expérimentées. Ainsi, Man and Nature a entrepris la recherche de partenaires et bailleurs pour prolonger le projet sur une nouvelle période triennale (20192022). L'enjeu est désormais d'entretenir, étendre et pérenniser la dynamique, grâce à la promotion et la commercialisation des produits aux échelles locale et sous-régionale. Ce nouveau projet en cours d'élaboration comporte en outre un important volet sur la gouvernance et le rôle des autorités locales dans un tel processus.

En effet, non seulement le potentiel de réplication est très important, mais les impacts positifs du modèle pourraient être encore renforcés en y intégrant les acteurs spécialisés dans la conservation - ONG ou gouvernements, qui ont la gouvernance des parcs. À l'avenir, il conviendra judicieusement d'exploiter les synergies fonctionnelle (développement durable/gouvernance) et territoriale (cœur/périphérie des AP) qui existent entre ces deux approches de protection de la biodiversité.

Enfin, ce cas d'étude et l'expérience de Man and Nature dans le développement de filières ont révélé qu'il est essentiel que les entreprises privées soient impliquées dès la conception du projet afin d'assurer en amont des débouchés et un transfert de compétences aux acteurs locaux sur la gestion de la ressource. En choisissant de s'associer à une telle approche, elles contribuent au développement durable des pays du Sud, et acquièrent de surcroît une meilleure maîtrise et traçabilité de leur sourcing, tout en appréciant concrètement les impacts positifs qu'elles ont sur la biodiversité et les communautés locales impliquées.

\section{Références}

AFNOR. 2015. Produits biosourcés - Analyse du cycle de vie. Norme Française NF EN 16760, décembre 2015, 33 p.

Arizmendi Gonzalez I. 2013. Quelle stratégie de gestion pour l'augmentation de la rentabilité des systèmes agroforestier: modélisation technico-économique des agroforêts cacaoyers et caféiers améliorés au Cameroun. Montpellier : CIHEAM-IAMM, 120 p. (Master of Science-2013; n ${ }^{\mathrm{o}} 125$ ).

Assembe Mvondo S, Lema Ngono D. 2007. Droits des populations locales et conservation des ressources forestières: analyse du cas du sanctuaire à gorilles de Mengame-Cameroun. J Droit
Environ Dév 270. Disponible sur http://www.lead-journal.org/ content/07270.pdf.

Badey L, Bocquelet H, Delaye N. 2017. Référentiel méthodologique pour la réalisation d'ACV dans le secteur des huiles végétalesVersion 4. ITERG, octobre 2017, 60 p.

CBFP. 2005. The forests of the Congo Basin: A preliminary assessment. CARPE, $33 \mathrm{p}$.

COMIFAC. 2013. Boîte à outils sur l'intégration du DAA dans le secteur des PFNL en Afrique centrale. FAO, 144 p.

Cruz JF, Troude F, Griffon D, Hébert JP. 1988. Les générateurs d'air chaud. In: Conservation des grains en régions chaudes. $2^{\mathrm{e}}$ éd. Paris : Ministère de la Coopération et du Développement, $545 \mathrm{p}$.

Diedhiou Y, Diawara B. 2015. Rapport de mission de suivi réactif conjointe UNESCO/UICN dans le parc national de la Réserve de Faune du Dja au Cameroun. UNESCO \& UICN, novembre 2015, $67 \mathrm{p}$.

Fodouop Kengné. 2003. Développement rural dans la province du centre au Cameroun. Cah d' O.-M. [en ligne], consulté le 11 octobre 2018. Disponible sur http://journals.openedition.org/com/930.

GWZ Wijma Cameroun S.A. 2011. Derrière le label FSC de Wijma Cameroun S.A. Newsletter no. 2, février 2011 [en ligne], consulté le 13/07/2018. Disponible sur https://pfbc-cbfp.org/documents. html.

Kwesseu Petguen JM. 2010. Analyse qualitative des systèmes de cacaoculture dans la région du centre, Cameroun. Mémoire de fin d'études en vue de l'obtention du Diplôme d'Ingénieur Agronome, Économies et Sociologies Rurales. Dschang: Faculté d'agronomie et des sciences agricoles, Université de Dschang, $115 \mathrm{p}$.

McNeely JA. 1994. Des zones protégées pour le $21^{\mathrm{e}}$ siècle : Améliorer leur utilité pour la société. Biodiversity and Conservation. DOI: $10.1007 / \mathrm{BF} 00057797$.

Mukam FAJ. 2017. Amélioration des moyens d'existence des ménages, perception de la faune et réduction du braconnage à la périphérie nord de la Réserve de Biosphère du Dja. Mémoire en vue de l'obtention du Diplôme d'ingénieur Agronome, Économies et Sociologies Rurales. Dschang: Faculté d'Agronomie et des Sciences Agricoles, Université de Dschang, 89 p. UnasylvaNo. 176. FAO, 1994.

Ndzomo Abanda G. 2008. Concilier droits des pygmées et gestion durable de la biodiversité. AGRIDAPE 24(3): 17-20.

Ngome-tata P. 2006. Étude sur la gestion durable des PFNL au Cameroun. IUCN, octobre 2006, $41 \mathrm{p}$.

Rothe PL. 1964. Régénération naturelle en forêt tropicale: le Dipterocarpus dyeri (Dau) sur le versant cambodgien du golfe du Siam. Bois For Trop 8: 386-397.

The Rainforest Alliance. 2017. Guide for the Sustainable Agriculture Standard. July 2017, Version 1.2, 122 p.

Citation de l'article : Roques E, Lachaux C, Tournebize T, Epanda AM, Mikam Akongongol M. 2019. Un modèle économique et social pour la conservation de la biodiversité : des filières oléagineuses durables issues de produits forestiers non ligneux (PFNL) pour préserver la Réserve de Faune du Dja (RFD) au Cameroun. OCL 26: 7. 